\title{
Resin Transfer Molding Process: A Numerical Investigation
}

\author{
I. Rodrigues de Oliveira ${ }^{1, a}$, S. Campos Amico ${ }^{2, b}$, J. Ávila Souza ${ }^{3, c}$, \\ F. Ferreira Luz ${ }^{2, d}$, R. Barcella ${ }^{2, e}$ and A. Gilson Barbosa de Lima ${ }^{1, f}$ \\ ${ }^{1}$ Department of Mechanical Engineering, Federal University of Campina Grande (UFCG), Zip Code \\ 58429-900, Campina Grande-PB, Brazil. \\ ${ }^{2}$ Department of Materials Engineering, Federal University of Rio Grande do Sul (UFRGS), Zip \\ Code 91501-970, Rio Grande do Sul-RS, Brazil. \\ ${ }^{3}$ Department of Mechanical Engineering, Federal University of Rio Grande (FURG), Zip Code \\ 96201-900, Rio Grande-RS, Brazil. \\ arodrigues.iran@hotmail.com, bamico@ufrgs.br, cjasouza@furg.br, dfelipe.luz@ufrgs.br, \\ 'rodbj1986@gmail.com, 'gilson@dem.ufcg.edu.br
}

Keywords: RTM, flow simulation, linear injection, pressure

\begin{abstract}
In the processing of high performance composite materials, the RTM process has been widely used by many sectors of the industry. This process consists in injecting a polymeric resin through a fibrous reinforcement arranged within a mold. In this sense, this study aims to simulate the rectilinear infiltration of pure resin and filled resin $\left(40 \% \mathrm{CaCO}_{3}\right)$ in a mold with glass fiber preform, using the PAM-RTM commercial software. Numerical results of the filling time and fluid front flow position over time were assessed by comparison with the experimental data and a good accuracy was obtained.
\end{abstract}

\section{Introduction}

Resin Transfer Molding (RTM) process consists in positioning the preform (i.e. fibrous reinforcement preform in the shape of the piece) within the mold, commonly glass fiber or carbon fiber. This preform is a dry porous/fibrous medium through which fluid flow occurs. The mold is closed and resin is injected into the mold cavity until the fibrous reinforcement is fully impregnated. After resin curing, the mold is opened and the part is removed from the mold. This part may still require finishing operations and/or to go through a post-curing process. This process has proved highly effective in the processing of high performance composite materials.

In the RTM process, there are some factors that should be known and controlled as the resin viscosity, injection pressure, content and arrangement of the fibers, porosity, process temperature and permeability of the porous media. The injection pressure and temperature gradient, for example, must be optimized so that the finishing end of the composite and productivity are suitable [1].

In addition to experimental studies, numeric analysis software have been frequently used to predict the resin behavior within the preform/mold along the RTM process, evaluate the filling time (which should be less than the resin's gel time), evaluate deficient impregnation points and determine the resin injection points and vent ports [2]. There are different software dedicated exclusively to study RTM process such as PAM-RTM from ESI Group, the RTM-WORX from Polywork and the LIMS from the University of Delaware which are commonly used by industry for having a simple usage and focused only on this process. The non-dedicated commercial software commonly used to study CFD (Computational Fluid Dynamics) are Ansys CFX ${ }^{\circledR}$ and FLUENT, both from ANSYS and Abaqus CFD from Simula Abaqus, which are simulation tools for fluid mechanics and heat transfer problems, capable of working with complex geometries and simulate the resin's advancement and curing within the mold. Several authors have studied the process of resin transfer molding, such as: Hiller et al. [3]; Amorim Jr. [4]; Machado et al. [5]; Garay [6]; Sánchez et al. [7]; Jiang et al. [8]; Luz et al. [9]; Rudd [10] and Luz et al.[11]. 
In complement for these cited studies, this work aims to simulate a rectilinear infiltration in a glass fiber mat fibrous media using the PAM-RTM software, and evaluate the influence of process parameters, such as resin viscosity, porous media permeability and $\mathrm{CaCO}_{3}$ content, at the resin infiltration velocity in the porous media.

\section{Methodology}

For the numeric results validation, experiments were performed injecting orthophthalic polyester resin in fiberglass mat for two different infiltration cases:

- Non-filled resin $\left(0 \% \mathrm{CaCO}_{3}\right)$, composite density $\rho=1190 \mathrm{~kg} / \mathrm{m}^{3}$, fiber volume fraction $\mathrm{V}_{\mathrm{f}}=$ $19 \%$, porosity $\varphi=0.8$;

- Filled resin with $40 \% \mathrm{CaCO}_{3}$, composite density $\rho=1500 \mathrm{~kg} / \mathrm{m}^{3}$, fiber volume fraction $\mathrm{V}_{\mathrm{f}}=$ $19 \%$, porosity $\varphi=0.81$.

In this work we use a mesh with dimensions $320 \times 150 \times 3.6 \mathrm{~mm}$. In the mold, the rectangular region is specially designed to facilitate the formation of the linear profile of the resin forward advancement. This mold outline is required to be able, in laboratory experiments, to determinate the porous media permeability from the experimental data. This mesh has 7077 elements and 3685 nodes, with better refinement in the zone near to the injection region.

In all simulatiois it was assumed an incompressible fluid flow through a porous medium. The numerical model used is based on the Darcy law, and is giver as fallows [1,10].

$$
\begin{aligned}
& \vec{v}=-\frac{\mathbf{i}}{\mu} k \nabla P \\
& \boldsymbol{\nabla} \cdot \vec{v}=\mathbf{0}
\end{aligned}
$$

where $\vec{v}$ is the fluid velocity vector $[\mathrm{m} / \mathrm{s}] ; k$ is the porous media permeability $\left[\mathrm{m}^{2}\right] ; \nabla \mathrm{P}$ is the pressure gradient $[\mathrm{Pa}]$ and $\mu$ is the resin viscosity (considered as a Newtonian fluid) [Pa s].

The model was solved using a non-conforming finite element approximation. The pressure is discontinuous along the inter-element boundaries except at the middle nodes, and filling factors are associated with the mesh elements [12].Table 1 summarizes the initial and boundary conditions for the studied cases.

Table 1 - Initial and boundary conditions for the studied cases.

\begin{tabular}{ll}
\hline Non-filled resin & Filled resin with $40 \% \mathrm{CaCO}_{3}$ \\
\hline $\mathrm{P}_{\text {inlet }} \rightarrow$ varying from 0 to 20300 & $\mathrm{P}_{\text {inlet }} \rightarrow$ varying from 0 to 21300 \\
$\mathrm{~Pa}$ (manometric) & $\mathrm{Pa}$ (manometric) \\
\hline Wall $\rightarrow$ zero pressure gradient & Wall $\rightarrow$ zero pressure gradient \\
\hline $\mathrm{P}_{\text {front flow }}=0 \mathrm{~Pa}$ (manometric) & $\mathrm{P}_{\text {front flow }}=0 \mathrm{~Pa}$ (manometric) \\
\hline $\mathrm{T}_{\text {resin,inlet }}=291.7 \mathrm{~K}$ & $\mathrm{~T}_{\text {resin,inlet }}=293.45 \mathrm{~K}$ \\
\hline
\end{tabular}

\section{Results and Discusions}

Table 2 shows results for viscosity and permeability as a function of calcium carbonate content and injection pressure. It is observed that the addition of $\mathrm{CaCO}_{3}$ modifies the permeability and increases the viscosity. It is realized that the higher the concentration of $\mathrm{CaCO}_{3}$ in the resin, the greater the permeability enhancement due to the inherent characteristics of the experimental method used for estimation of this parameter (rectilinear flow) and so varying the properties of the fibrous reinforcement.

Table 2 - Viscosity and permeability data for each experiment.

\begin{tabular}{cccc}
\hline & Viscosity $(\mathrm{cP})$ & Maximum injection pressure (bar) & Permeability $\left(\mathrm{x}^{-10} \mathrm{~m}^{2}\right)$ \\
\hline $0 \% \mathrm{CaCO}_{3}$ & 330 & 0.203 & 6.6 \\
$40 \% \mathrm{CaCO}_{3}$ & 2113 & 0.213 & 1.19 \\
\hline
\end{tabular}


With the increasing in fluid viscosity, increases the filling time, i.e. the time for completion the fibrous media with resin, as show in Table 3, where the experimental results are compared with the PAM-RTM numeric solution.

Table 3 - Filling time for experimental and numeric results.

\begin{tabular}{cccc}
\hline & \multicolumn{3}{c}{ Filling time (s) } \\
& Experimental & Numerical & Error \\
\hline $0 \% \mathrm{CaCO}_{3}$ & 883 & 994 & $12.57 \%$ \\
$40 \% \mathrm{CaCO}_{3}$ & 3420 & 3410 & $0.20 \%$ \\
\hline
\end{tabular}

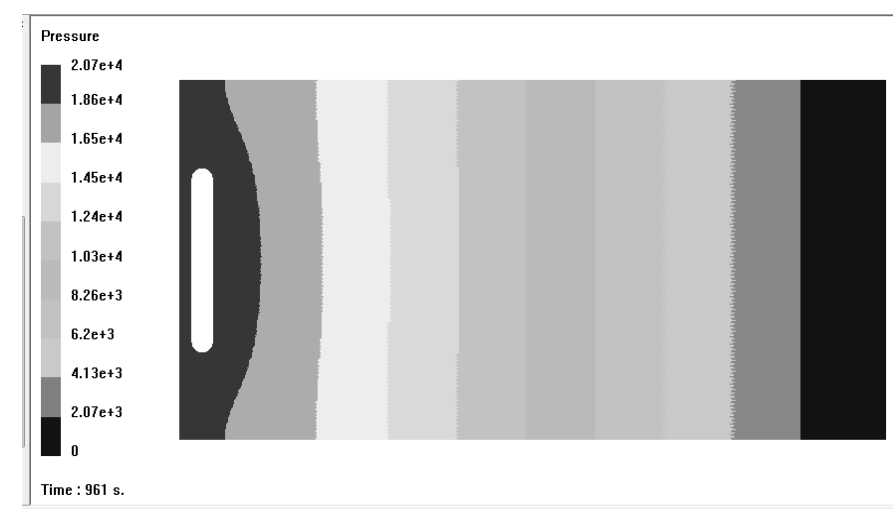

a) $\mathrm{t}=961 \mathrm{~s}\left(0 \% \mathrm{CaCO}_{3}\right)$

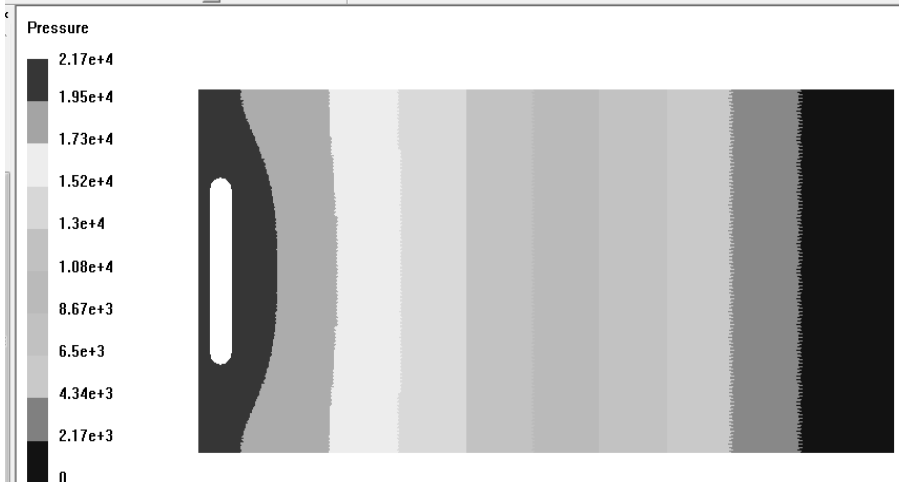

b) $\mathrm{t}=3150 \mathrm{~s}\left(40 \% \mathrm{CaCO}_{3}\right)$

Figure 1- Pressure field obtained with PAM-RTM.

Figs. 1 and 2 illustrate the pressure behavior within the preform for the filling times $t=961 \mathrm{~s}$ $\left(0 \% \mathrm{CaCO}_{3}\right)$ and $\mathrm{t}=3150 \mathrm{~s}\left(40 \% \mathrm{CaCO}_{3}\right)$, respectively. It is perceived that the injection pressure obtained by the PAM-RTM numeric solution approaches the experimental results obtained for both cases. We can see that the higher pressure occurs of the injection part and lower pressure is verified in the vent part, as expected, because maximum and minimum pressures correspond to boundary conditions for the studied physical problem.

The flow front advance in the $0 \% \mathrm{CaCO}_{3}$ case during the resin injection is shown in Fig.2. The rectilinear flow front profile has occurred at approximately half length of the mold. Initially, at the region close to the injection port, the flow has 2D characteristics and the flow front assumes a ring (radial) shape form in the main flow direction. When the pressure gradient become linear, the flow front tends to become rectilinear (1D).

To validate the simulation obtained with PAM-RTM, the results were compared with experimental data, as shown in Figs. 3, 4 and 5. Detail about the equipment and experimental procedure can be found in [1, 6, 10,11]. Fig. 3 shows the experimental flow front positions along the fiber media for different processing times. Fig. 4 shows the experimental and numerical flow 
front positions as a function of time for the case $\left(40 \% \mathrm{CaCO}_{3}\right)$. Results show that the PAM-RTM numerical solution is in good agreement with experimental results. A large error is observed in the nonlinear (close to the injection section) region. The difference is probably due to the 3D characteristic of the experimental setup on which the injection is performed through the bottom of the mold while in the numerical solution prescribed pressure is specified at the borders of the injection hole. Besides, the permeability was determined based or the $1 \mathrm{D}$ rectilinear flow $(\mathrm{t}>100 \mathrm{~s})$ $[1,6,9,10]$.

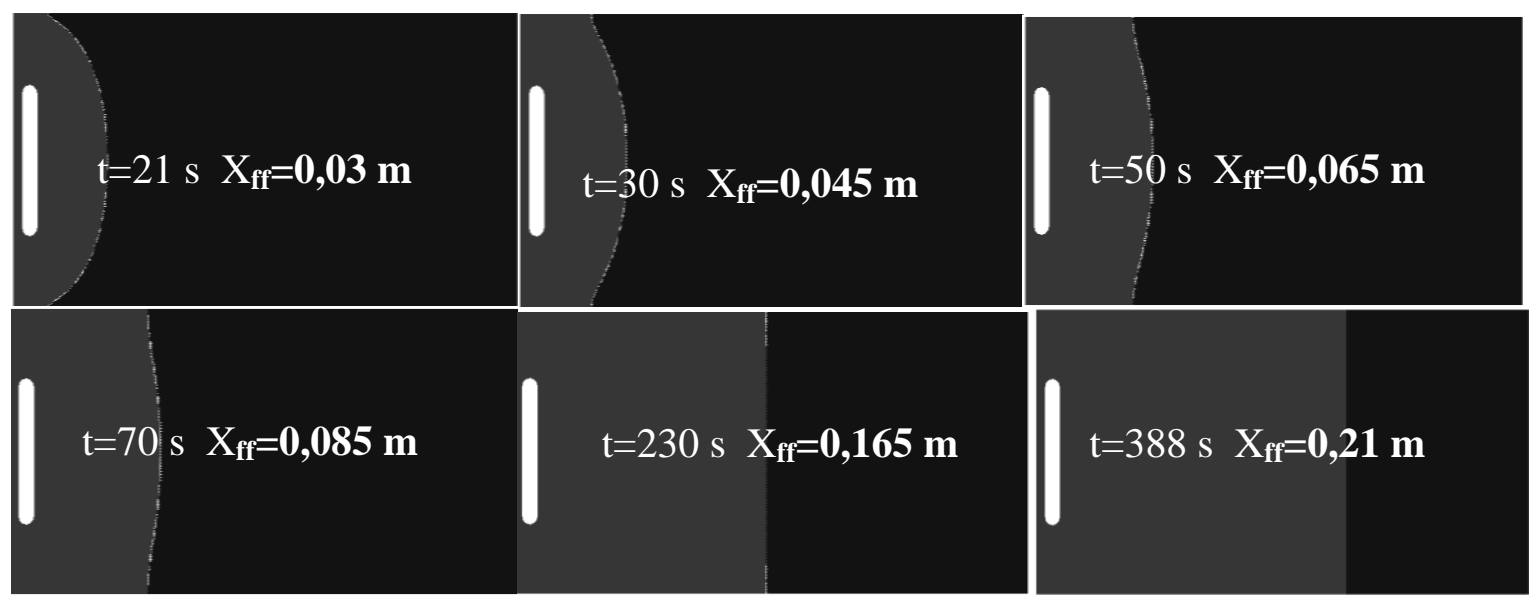

Figure 2 - PAM-RTM simulation for the front flow in the case $\left(0 \% \mathrm{CaCO}_{3}\right)$ at different times.

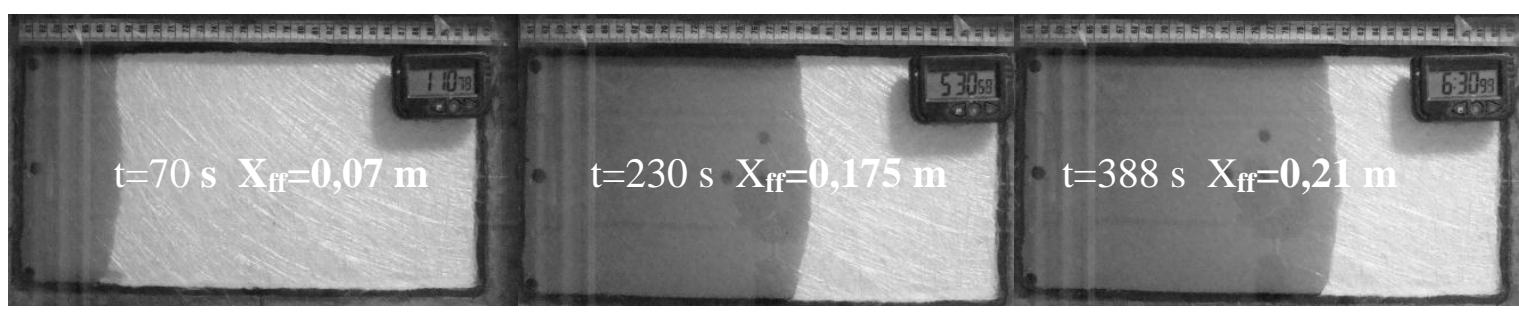

Figure 3 - Experimental front flow in the non-filled resin case $\left(0 \% \mathrm{CaCO}_{3}\right)$ at different times.

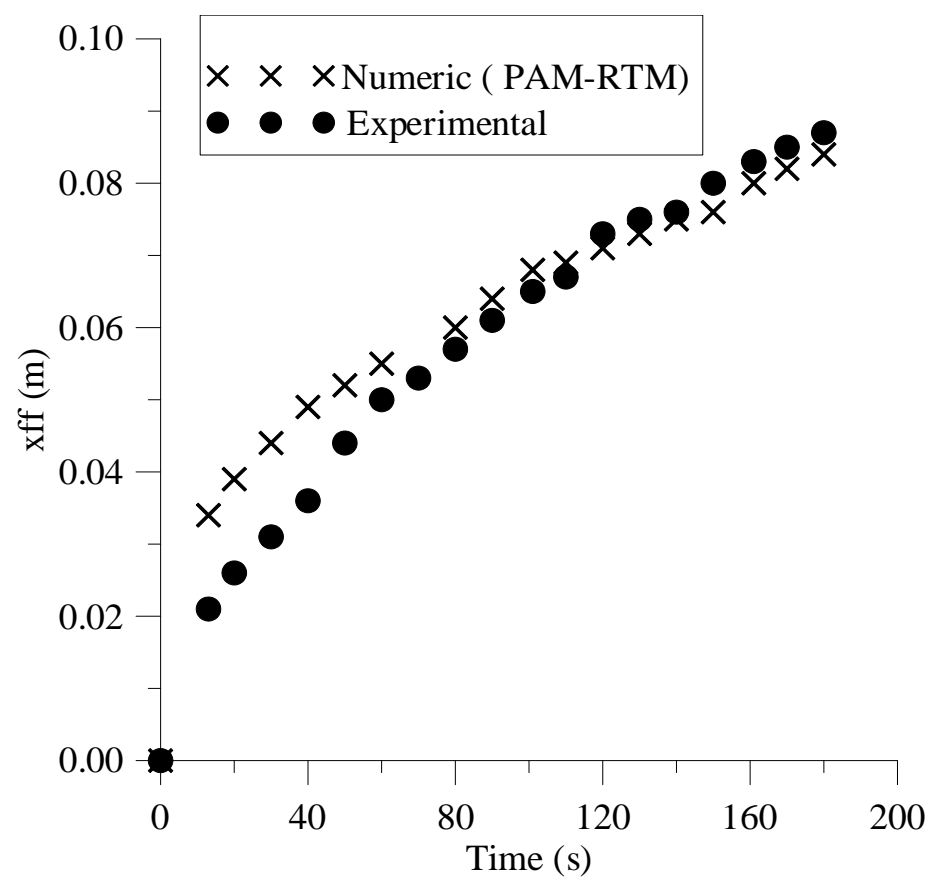

Figure 4 - Resin position versus time for the case $40 \% \mathrm{CaCO}_{3}$. 


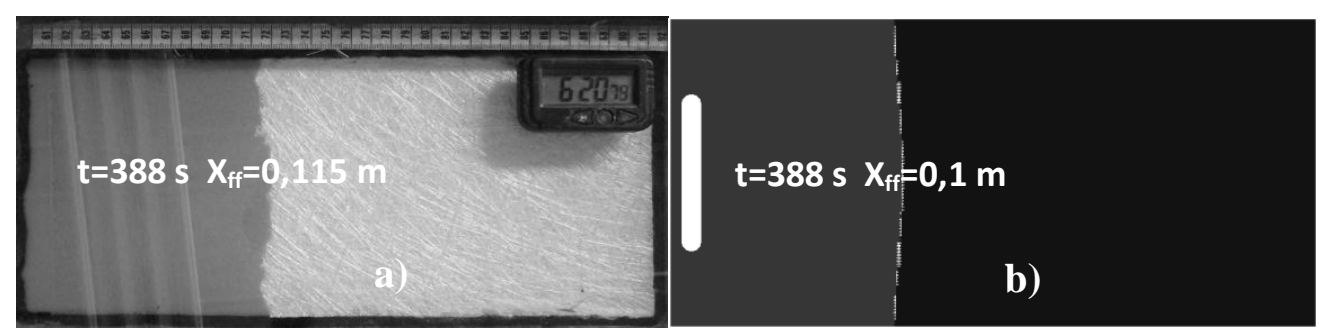

Figure 5 - Comparative of the experimental data (a) and PAM-RTM solution (b) for the fluid flow front for the filled resin case $\left(40 \% \mathrm{CaCO}_{3}\right)$.

Comparing the PAM-RTM results with the experimental data for the time $388 \mathrm{~s}$ for the filled resin $\left(40 \% \mathrm{CaCO}_{3}\right)$ and non-filled resin cases (Figs. 2, 3 and 5), we can be observe the effect of adding calcium carbonate in the filling time, the higher the $\mathrm{CaCO}_{3}$ concentration in the resin, the bigger the filling time. This is due to the presence of calcium carbonate particles among the fibers, hindering the resin flow through the fibrous media.

\section{Conclusions}

This paper provides numerical and experimental information about RTM process. The PAM-RTM commercial software has been applied to simulate resin flow in porous fibrous.

Through the numerical and experimental results it can be concluded that:

a) The computational model represented well the physics of the problem, it was possible to simulate the total resin filling time for both non-filled $\left(0 \% \mathrm{CaCO}_{3}\right.$ and filled resin $(40 \%$ $\mathrm{CaCO}_{3}$ ) cases and predict the fluid flow front profile in the mold.

b) Addition of $\mathrm{CaCO}_{3}$ amending the permeability values, increases viscosity and the filling time. The numerical results showed good agreement with the experimental data in terms of front flow position, filling time and injection pressure.

\section{Acknowledgements}

The authors thank to CNPq and CAPES for financial support and to authors referred to their research that contributed in this improvement of this work.

\section{References}

[1] F.F. Luz: Comparative Analysis of Fluid Flow in Experiments Using RTM Commercial Applications (MSc. Thesis. Federal University of Rio Grande do Sul, Brazil, 2011). (In Portuguese)

[2] R. Stewart: Reinf. Plast., Vol.26 (2002), p. 46.

[3] G.R. Hiller, J.L. Filla Jr, T.M. Schmidt and S.C. Amico: XVII CBECIMat - Brazilian Congress of Engineering and Material Sciences, 2006, Foz do Iguaçu, Brazil.

[4] W.F. Amorim Jr.: Processing the Composite Thick Plate by Resin Transfer Molding Applications (MSc Thesis, Federal University of Rio Janeiro, Rio de Janeiro, Brazil, 2007). (In Portuguese).

[5] G.L.P. Machado, A.C. Garay, P.B. Roese, T. M. Schmidt, J. A. Souza and S. C. Amico: Numerical Simulation of Infiltration in Manufacturing Process for Composite RTM. X CBpolBrazilian Congress of Polymers, 2009, Foz do Iguaçu, Brazil.

[6] A.C. Garay, V. Heck, A.J. Zattera, J.A. Souza and S.C. Amico: J. Reinf. Plast. Comp. vol. 30 (2011), p. 1213 
[7] F. Sánchez, J. A. García, L. Gascón and F. Chinesta: Comput. Meth. Appl. Mech. Eng. Vol.196 (2007), p. 2300

[8] S. Jiang, L. Yang, S. Alsoliby and G. Zhou: Comp. Sci. Technol., Vol.67 (2007), p.3316-3322.

[9] F.F. Luz, S.C. Amico, A.L. Lima, E.S. Barbosa and A.G.B. Lima: Defect Diffusion Forum, Vol 326-328 (2012).

[10]C.D. Rudd, K.N. LAC and C.G.E. Mangin: Liquid Moulding, Structural Reaction Injection Moulding and Related Processing Techniques (Woodhead, Publishing Limited, 1997).

[11]F.F. Luz, S.C. Amico, J.A. Souza, E.S. Barbosa and A.G.B. Lima, in: Numerical Analysis of Heat and Mass Transfer in Porous Media, edited by J.M.P.Q. Delgado, Antonio Gilson Barbosa de Lima and Marta Vázquez da Silva, Springer, Heidelberg (2012).

[12]PAM-RTM - User`s Guide \& Tutorials, 2009. 
Diffusion in Solids and Liquids VIII

10.4028/www.scientific.net/DDF.334-335

Resin Transfer Molding Process: A Numerical Investigation

10.4028/www.scientific.net/DDF.334-335.193 